\title{
Annuncio Congresso
}

\section{May-2 June 2002}

$5^{\text {th }}$ ENMESH (European Network for Mental Health Service Evaluation) Conference “MENTAL HEALTH FOR A UNITED EUROPE: SERVICE RESEARCH IN CONTEXT ${ }^{n}$ - Sofia (BG)

\section{Conference topics:}

- Stigma, discrimination and equity;

- Knowledge transfer and service culture;

- Economic issues in psychiatry across Europe;

- Evaluating mental health policies.

In addition training events will be held. Simultaneous translation into Russian will be provided.

ENMESH is a network of more than 200 professionals from more than 20 European countries in the field of mental health services research. The ENMESH Executive Committee:

OM. Knapp, UK, H.C. Knudsen, DK, A. H. Schene, NL, M. Tansella, I, G. Thornicroft, UK, J.L. Vazquez-Barquero, SP.

Local Organizing Committee: T. Tomov (Chair), M. Okoliyski, V. Hristakeva. Info: Association of reformers in psychiatry, 1 Maliovica str., 1001 Sofia, Bulgaria Tel: +359 9877875; e-mail: arp@inet.bg www.enmesh2002.org 


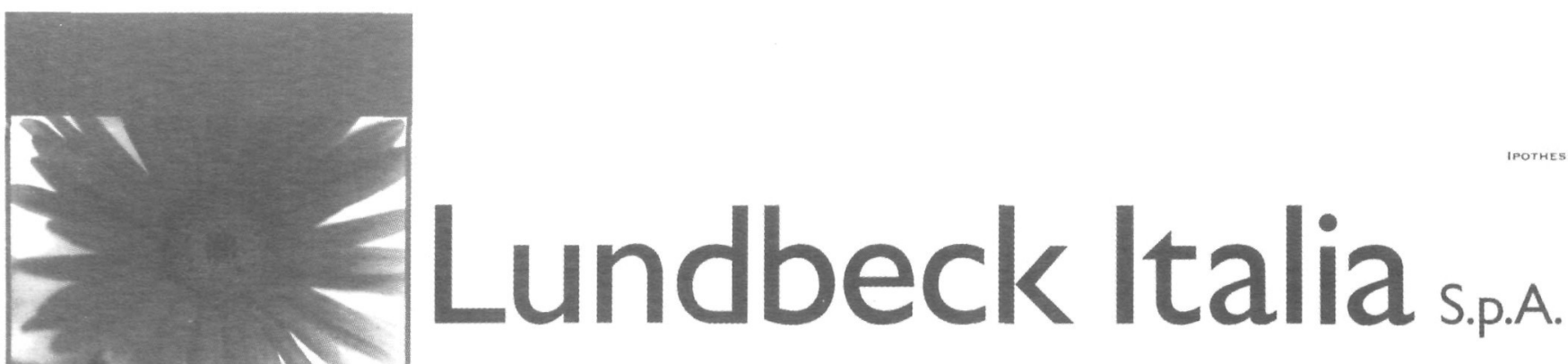

\section{Sezione per il Medico}

L'accesso è subordinato al possesso di una password fornita dall'Azienda.

\section{Congressi}

La rubrica, aggiornata mensilmente, è articolata in 3 sezioni:

- congressi patrocinati da Lundbeck Italia

- congressi in "diretta"

- calendario congressi

\section{Domande e Risposte}

I quesiti inviati via e-mail dai medici saranno vagliati e selezionati dall'esperto, oppure dalla redazione e poi proposti all'esperto. Alle domande più interessanti l'esperto risponderà pubblicamente nella rubrica.

\section{Eventi on-line}

Questo spazio di discussione aperto alla partecipazione dei visitatori del sito, sarà organizzato per argomenti specifici, in diretta con la partecipazione di un esperto.

\section{Mailing List}

Lista di discussione via e-mail sempre aperta agli iscritti. Non sarà strettamente moderata, ma guidata. Un esperto, riconosciuto come gestore della lista, potrà proporre argomenti, stimoiare e dirigere la conversazione.

\section{Professione: specialista S.N.C.}

Informazioni e consigli per la professione (consenso informato, normativa, responsabilità...)

\section{Lundbeck per il medico}

-Versione on-line di "Noos", aggiornamenti in psichiatria

- I progetti educazionali: "Dedalo" e "Medstar"

- Indirizzi e nominativi degli ISF Lundbeck Italia

- Il listino Lundbeck

\section{Bibliografia e Medline}

- Selezione internazionale specifica

- Siti istituzionali Lundbeck

\section{News}

Notizie specifiche sulla psichiatria/neurologia con "spigolature" dalla letteratura scientifica.

\section{WW
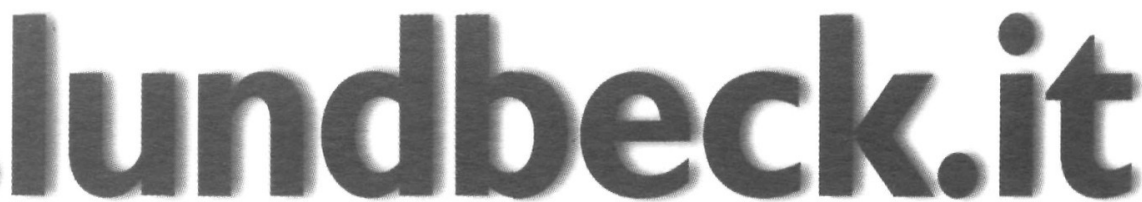

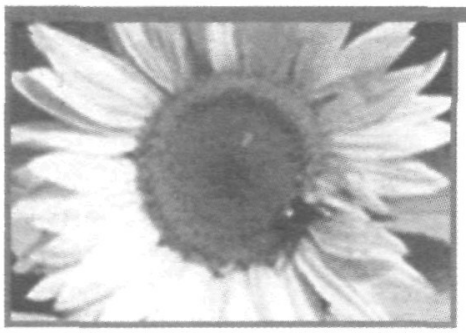

\section{L'Azienda}

Azienda: la nostra "visione" - la nostra identità - Lundbeck nel mondo. Tutte le informazioni su Lundbeck Italia.

\section{Consigli Pratici}

Informazioni su condizioni come disturbi dell'umore, schizofrenia, ecc. I sintomi, la diagnosi, gli specialisti a cui rivolgersi, le terapie (con collegamento alla sezione riservata ai medici per la descrizione dei farmaci).

\section{Sezione aperta al Pubblico}




\section{Articoli \\ 260}

Le strutture residenziali psichiatriche in Italia. I risultati della fase I del progetto PROGRES

276

Monitorare l'impatto sulla letteratura internazionale della produzione scientifica dei ricercatori italiani, nelle discipline "psichiatria» e "psicologia». Quinquiennio 1995-1999 e confronto con il 1985-1989 M. Tansella, G.A. Fava

283

Elenco degli Autori di Invited Editorials (1992-200I)

284

Elenco degli Autori

di Invited Papers (1992-200I)

285

Indice generale del volume 10 (200I) 